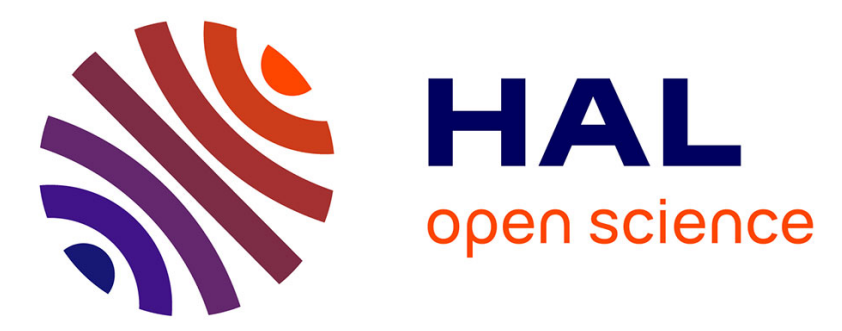

\title{
The Exact Intuitionistic Meaning of the Square of Opposition
}

\author{
Joseph Vidal-Rosset
}

\section{To cite this version:}

Joseph Vidal-Rosset. The Exact Intuitionistic Meaning of the Square of Opposition. Jean-Yves Béziau, Gianfranco Basti The Square of Opposition: A Cornerstone of Thought, Springer International Publishing, pp.201-303, 2017, Studies in Universal Logic, 978-3-319-45061-2 (Print) 978-3-319-45062-9 (Online). 10.1007/978-3-319-45062-9_17 . hal-01534557

\section{HAL Id: hal-01534557 https://hal.science/hal-01534557}

Submitted on 15 Jun 2017

HAL is a multi-disciplinary open access archive for the deposit and dissemination of scientific research documents, whether they are published or not. The documents may come from teaching and research institutions in France or abroad, or from public or private research centers.
L'archive ouverte pluridisciplinaire HAL, est destinée au dépôt et à la diffusion de documents scientifiques de niveau recherche, publiés ou non, émanant des établissements d'enseignement et de recherche français ou étrangers, des laboratoires publics ou privés.

\section{(이) $\$$}

Distributed under a Creative Commons Attribution - NonCommercial - NoDerivatives| 4.0 


\title{
The Exact Intuitionistic Meaning of the Square of Opposition
}

\author{
Joseph Vidal-Rosset
}

To David DeVdi and to Sean McLaughlin

\begin{abstract}
This paper aims at providing a complete analysis of the intuitionistic version of the square of opposition and a reply to an article published by Mélès (Around and Beyond the Square of Opposition, ed. by J.-Y. Béziau, D. Jacquette (Studies in Universal Logic, Birkhaüser, 2012), pp. 201-218) on the same topic.
\end{abstract}

Keywords Intuitionistic logic - Square of opposition - Tableau methods

Mathematics Subject Classification (2000) Primary 03B20, 03F03; Secondary 03B10

\section{The Classical Square of Opposition}

I assume that the reader is familiar both with classical first order logic (for short, CFOL) and the standard symbolism adopted in this paper. Showing the discrepancies between, on the one hand, the square of opposition in CFOL and, on the other hand, the intuitionistic first order logic (for short, IFOL) is a good way to understand the latter; but before one must grasp the former. The well known classical square of opposition i.e. Fig. 1, page 292 is constructed by superimposing the two polygons of Fig. 2, page 292. Each valid implication in these polygons gives the meaning of what is either a subaltern proposition, or a contrary proposition, or contradictory proposition, or a sub-contrary proposition.

Definition 1.1 I call "negative" any formula obtained by prefixing one negation symbol to a formula of the square, i.e. $\neg(A), \neg(E), \neg(I), \neg(O)$. A formula is "positive" if it is not negative: (A), (E), (I), (O) are positive.

Remark 1.2 There is no valid implication between two negative formulas, and there is only one validity only between a couple of positive formulas, i.e. in the subaltemation case.

Remark 1.3 Most valid implications contain a positive and a negative formula.

Every theorem of this paper has been checked by IMOGEN i.e. Sean McLaughlin' theorem prover for intuitionistic First Order Logic (https://github.com/seanmcl/imogen). 


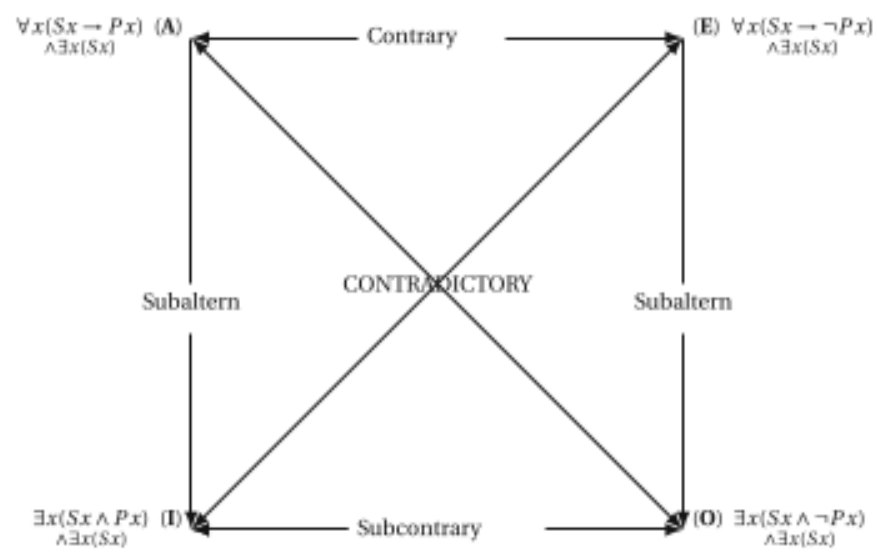

Fig. 1 Classical square of opposition
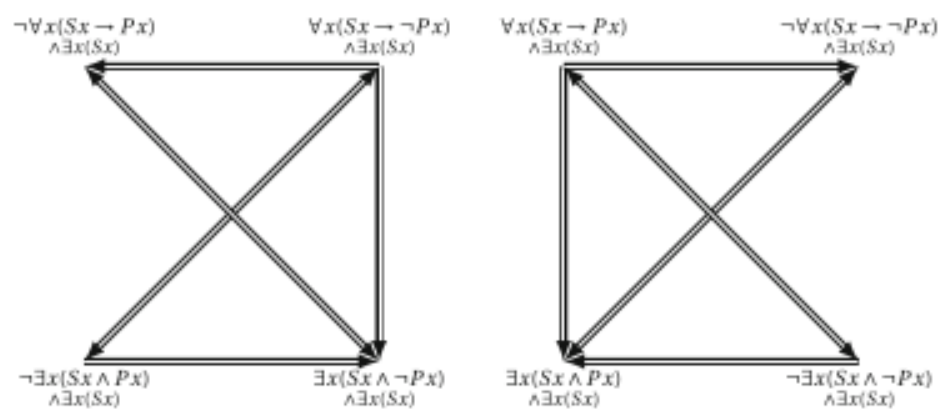

Fig. 2 Implications valid in CFOL

Remark 1.4 Contrariety and sub-contrariety relations are duals of each other: the former assumes a positive formula and concludes on a negative consequence, while the latter assumes a negative formula and concludes on a positive consequence. That explains why it is said, to explain the contrariety relation, that (A) and (E) cannot be both true but can be both false, and that $(\mathrm{I})$ and $(\mathrm{O})$ cannot be both false but can be both true, to explain the sub-contrariety relation.

Remark 1.5 The contradiction relation define four equivalences in the square, i.e.

1. (A) $\leftrightarrow \neg(O)$

2. $(O) \leftrightarrow \neg(A)$

3. $(E) \leftrightarrow \neg(I)$

4. $(I) \leftrightarrow \neg(E)$ 
Remark 1.6 The existential assumption $\exists x(S x)$ is not necessary to prove a contradiction relation in the square; one needs the following equivalences to do it ${ }^{1}$ :

$$
\begin{gathered}
(\neg \neg A A) \wedge(A \rightarrow \neg \neg A) \\
(\neg \forall x \neg(F x) \rightarrow \exists x(F x)) \wedge(\exists x(F x) \rightarrow \neg \forall x \neg(F x)) \\
(\neg \exists x \neg(F x) \rightarrow \forall x(F x)) \wedge(\forall x(F x) \rightarrow \neg \exists x \neg(F x)) \\
((A \rightarrow B) \rightarrow(\neg A \vee B)) \wedge((\neg A \vee B) \rightarrow(A \rightarrow B)) \\
(\neg(A \wedge B) \rightarrow(\neg A \vee \neg B)) \wedge((\neg A \vee \neg B) \rightarrow \neg(A \wedge B)) \\
(\neg(A \vee B) \rightarrow(\neg A \wedge \neg B)) \wedge((\neg A \wedge \neg B) \rightarrow \neg(A \vee B))
\end{gathered}
$$

\section{Example 1.7}

$$
\vdash_{c} \neg \forall x(S x \rightarrow P x) \leftrightarrow \exists x(S x \wedge \neg P x)
$$

Proof A simple proof can be made on the basis of the classical equivalences above. By (1.3) and (1.4) one gets:

$$
\neg \forall x(S x \rightarrow P x) \leftrightarrow \neg \neg \exists x \neg(\neg S x \vee P x)
$$

By (1.1) and (1.6) one gets:

$$
\neg \neg \exists x \neg(\neg S x \vee P x) \leftrightarrow \exists x(\neg \neg S x \wedge \neg P x)
$$

By (1.1) one gets:

$$
\exists x(\neg \neg S x \wedge \neg P x) \leftrightarrow \exists x(S x \wedge \neg P x)
$$

\footnotetext{
${ }^{1}$ In this list, only (1.6) is an equivalence in IFOL: except this latter, all conjunctions, from (1.1) to (1.5) have a left component provable in CFOL but not in IFOL.
} 
By transitivity of $\leftrightarrow$, from (1.8) to (1.10), one gets the conclusion:

$$
\neg \forall x(S x \rightarrow P x) \leftrightarrow \exists x(S x \wedge \neg P x)
$$

\section{The Intuitionistic Square of Opposition}

\subsection{Bell-DeVidi-Solomon Proof Method for Intuitionistic First Order Logic}

To understand the tree proof method for intuitionistic logic used in this paper, I reproduce in Table 1 the tree rules given by Bell et al. [1, p. 197, pp. 216-27], and also the transport and closure rules ${ }^{2}$ :

\subsection{Mèlès' Incomplete Intuitionistic Square}

In a paper published in a volume edited by Béziau and Jacquette [2], Mélès [4, p. 207] writes that "in intuitionistic logic, the square is clearly incomplete" and illustrates immediately his claim by a figure that we reproduce in this paper in Fig. 3, page 295.

Unfortunately, Fig. 3 is misleading because it gives the impression that there is no square of opposition in IFOL. As we are going to see, Fig. 3 misses four provable implications in IFOL.

- First, in Fig. 3 the contradiction arrow from (A) to (O) must be restored. Indeed the implication

$$
(A \wedge B) \rightarrow \neg(\neg A \vee \neg B)
$$

is valid in intuitionistic logic, and also $\neg \exists x \neg(A x)$ is intuitionistically deducible from $\forall x(A x)$, and therefore ${ }^{3}$ :

$$
\vdash_{i} \forall x(S x \rightarrow P x) \rightarrow \neg \exists x(S x \wedge \neg P x)
$$

\footnotetext{
${ }^{2}$ I do not mention the fork rule, because it is not used in this paper. I strongly recommend the reading of Bell et al.'s book.

${ }^{3}$ Symbols $\vdash_{c}, \quad \nvdash_{c}, \quad \vdash_{i}, \quad \nvdash_{i}$ mean respectively: prowable in classical logic, unprovable in classical logic, prowable in intuitionistic logic, unprovable in intuitionistic logic. I use in this paper the algorithm of refutation trees defined by Bell, DeVidi and Solomon for intuitionistic logic [1, pp. 192-223] (see Sect. 2.1).
} 
The Exact Intuitionistic Meaning of the Square of Opposition

Table 1 Bell et al. tree rules for IFOL

Fig. 3 Mélès' incomplete intuitionistic square

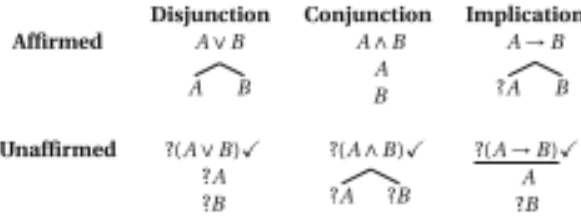

$$
\begin{aligned}
& \text { Affirmed } \begin{array}{cc}
\text { Equivalence } & \text { Negation } \\
A-B & 7 A \\
A \quad ? A & ? A \\
B \quad ? B &
\end{array} \\
& \text { Unaffirmed } \overbrace{?(A \rightarrow B)}^{P(A-B) \checkmark} \frac{\beta \rightarrow A \gamma}{A} \\
& \text { rule IUI rule IEI } \\
& \begin{array}{cc}
\forall x(F x) & \exists x(F x) \checkmark \\
F c & F c \\
\text { ny appropriate } c) & (c \text { new })
\end{array} \\
& \text { rule ?UI rule ?EI } \\
& \begin{array}{cc}
\frac{F \forall x(F x) \checkmark}{F c} & P \exists x(F x) \\
\langle c \text { new) } & F c \\
\text { (for any appropriate } c)
\end{array}
\end{aligned}
$$

Transport rule: We are allowed to carry any statement not marked by "?' across any horizontal line (i.e. locality) introduced by the ? $\rightarrow$ and ? $\rightarrow$ rules.

Checkmark: Any checkmarked formula is deactivated.

Closure rule. A path is closed when (and only when) both $P$ and $2 P$ occur on it not separated by a horizontal line. When it is the case, the path is marked by $X$. If all paths of a refutation tree $\mathscr{F}$ of a formula $F$ are closed, $\mathscr{F}$ is closed and that shows that the assumption of the intuitionistic unprovability of $F$ is a contradiction and that, therefore, $F$ is intuitionistically valid.
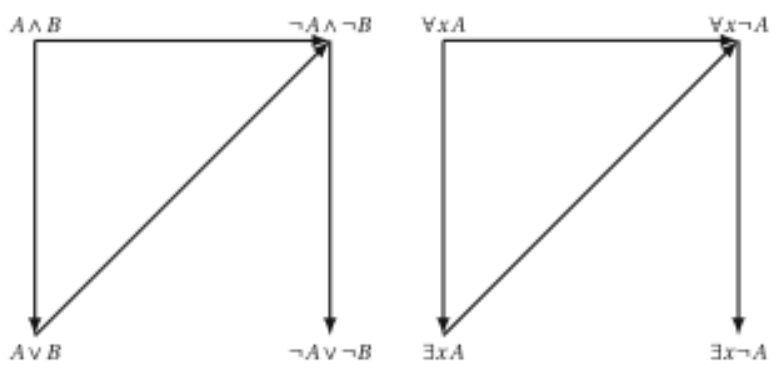
Proof

$$
\begin{gathered}
\frac{?(\forall x(S x \rightarrow P x) \rightarrow \neg \exists x(S x \wedge \neg P x))}{} \checkmark \\
\frac{? \neg \exists x(S x \rightarrow P x)}{\exists x(S x \wedge \neg P x) \checkmark} \\
S a \\
\neg P a \\
? P a \\
\forall x(S x \rightarrow P x) \\
\sim \\
? S a \quad P a \\
\times \quad \times
\end{gathered}
$$

- Second, if one pays attention to the other contradiction relation of the square, i.e. between (I) and (E), Fig. 3 is correct but incomplete: it is true that $(A \vee B)$ entails $\neg(\neg A \wedge \neg B)$ and that the converse is not intuitionistically provable. But Fig. 3 does not show that an equivalence is intuitionistically provable between $\neg(A \vee B)$ and $(\neg A \wedge \neg B)$ and also between $\neg(\mathrm{I})$ and $(\mathrm{E})$, i.e.

$$
\vdash_{i} \neg x(S x \wedge P x) \leftrightarrow \forall x(S x \rightarrow \neg P x)
$$

Proof

$$
\begin{aligned}
& \stackrel{?(\neg \exists x(S x \wedge P x) \leftrightarrow \forall x(S x \rightarrow \neg P x))}{ } \\
& \neg \exists x(S x \wedge P x) \quad \forall x(S x \rightarrow \neg P x) \\
& \frac{? \forall x(S x \rightarrow \neg P x) \checkmark}{S a} \quad \frac{? \neg \exists(S x \wedge P x) \checkmark}{\exists x(S x \wedge P x) \checkmark} \\
& \frac{? \neg P a}{P a} \\
& \neg \exists x(S x \wedge P x) \\
& ? \exists x(S x \wedge P x) \\
& \sim \\
& \text { ?Sa } \neg \mathrm{Pa} \\
& \times \quad ? P a \\
& \text { ?Sa } \quad ? \mathrm{~Pa} \\
& \mathrm{Sa} \times \\
& x
\end{aligned}
$$


- Third, according to Fig.3, the contrariety relation would be provable in intuitionistic logic only in the case of the implication $(A) \rightarrow \neg(E)$; $(E) \rightarrow \neg(A)$ would be intuitionistically unprovable. The following tree both refutes such a claim and proves this contrariety relation in IFOL:

$$
\vdash_{i}(\forall x(S x \rightarrow \neg P x) \wedge \exists x(S x)) \rightarrow \neg \forall x(S x \rightarrow P x)
$$

Proof

$$
\begin{aligned}
& \frac{?((\forall x(S x \rightarrow \neg P x) \wedge \exists x(S x)) \rightarrow \neg(\forall x(S x \rightarrow P x)))}{\forall x(S x \rightarrow \neg P x)} \\
& \exists x(S x) \checkmark \\
& \mathrm{Sa} \\
& \frac{? \neg(\forall x(S x \rightarrow P x))}{\forall x(S x \rightarrow P x)} \\
& \overbrace{}^{S a} \\
& \stackrel{\mathrm{Sa}}{\mathrm{Pa}} \\
& \text { ?Sa } \neg \mathrm{Pa} \\
& \times \quad P P a \\
& \times
\end{aligned}
$$

- Fourth, sub-contrariety $\neg(I) \rightarrow(O)$ is provable in intuitionistic propositional logic as well as in $\mathrm{IFOL}^{4}$ :

$$
\begin{gathered}
\neg(A \vee B) \rightarrow(\neg A \wedge \neg B),(\neg A \wedge \neg B) \rightarrow(\neg A \vee \neg B) \vdash_{i} \neg(A \vee B) \rightarrow(\neg A \vee \neg B) \\
\vdash_{i}(\neg \exists x(S x \wedge P x) \wedge \exists x(S x)) \rightarrow \exists x(S x \wedge \neg P x)
\end{gathered}
$$


Proof

$$
\begin{aligned}
& \frac{?((\neg \exists x(S x \wedge P x) \wedge \exists x(S x)) \rightarrow \exists x(S x \wedge \neg P x))}{\exists x(S x) \checkmark} \\
& \mathrm{Sa} \\
& \neg \exists x(S x \wedge P x) \\
& ? \exists x(S x \wedge \neg P x)
\end{aligned}
$$

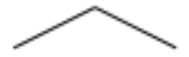

$$
\begin{aligned}
& \begin{array}{c}
2 S a \\
\times
\end{array} \frac{? \neg P a \checkmark}{P a} \\
& \mathrm{Sa} \\
& \neg \exists x(S x \wedge P x) \\
& P \exists x(S x \wedge P x) \\
& \curvearrowright \\
& \text { ?Sa } \quad ? \mathrm{~Pa} \\
& \times \quad \times
\end{aligned}
$$

Consequently, we can assert a positive theorem in the next section.

\subsection{A Proof That the Intuitionistic Square of Opposition Exists}

Theorem 2.1 The set of all implications intuitionistically provable in the classical square of opposition still defines a square.

Proof By superimposing the polygons of Fig. 4. Note that all contrariety and contradictory relations with positive assumptions and negative consequences are provable as well as in the classical case, and because one sub-contrariety relation remains provable in IFOL, a square of opposition exists in IFOL.

\subsection{Do We Lose Something with the Intuitionistic Version of the Square?}

As Fig. 4 shows, in IFOL the contradiction relations can no longer be expressed via the equivalences of Remark 1.5, except 3:

$$
\vdash_{i} \neg \exists x(S x \wedge P x) \leftrightarrow \forall x(S x \rightarrow \neg P x)
$$


The Exact Intuitionistic Meaning of the Square of Opposition
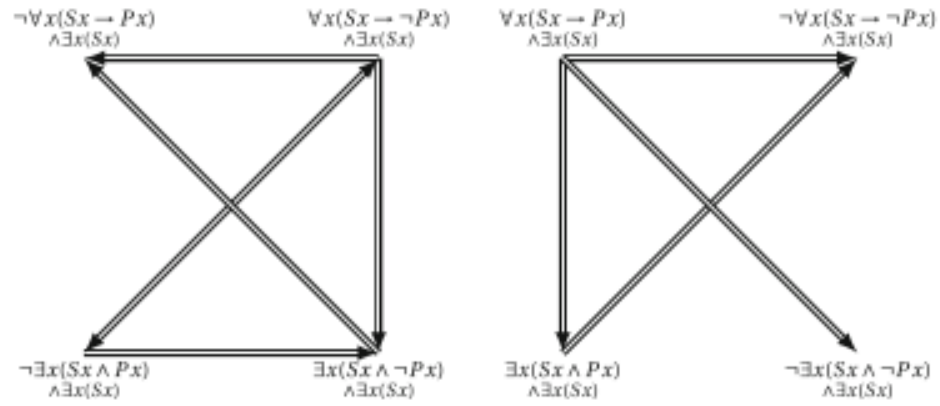

Fig. 4 Intuitionistic implications constructing the square

Comparing Figs. 4 and 2, it is clear that the beautiful duality of the classic square of opposition no longer exists in intuitionistic logic. So, in the polemic about classical logic vs. intuitionistic logic, we have to wonder if the square of opposition can be used as an argument on behalf of the conservative position. I am going to show that, in spite of the strength of classical logic, the reply to this question is negative.

One of the criticisms made against intuitionistic logic is that, being weaker than classical logic, intuitionistic logic prohibits some logical inferences as common as useful. When Mélès writes that there is an "intuitionistic gap in contradiction" [4, p. 208], it sounds like a similar reproach: it suggests that there are contradictory formulas in classical logic which are not identified as such in intuitionistic logic. ${ }^{5}$ It is indeed the case and it is provable as follows.

Definition 2.2 A formula $F$ is contradictory in FOL if and only if

$$
F \vdash \perp
$$

Theorem 2.3 There are contradictory formulas in CFOL that cannot be considered as such in IFOL

Proof In the classical square of opposition, the implication $\neg(A) \rightarrow(O)$ is provable, therefore its negation is a contradiction, i.e.

$$
\neg(\neg \forall x(S x \rightarrow P x) \rightarrow \exists x(S x \wedge \neg P x)) \vdash_{c} \perp
$$

but in IFOL, Glivenko's theorem fails and the double negation of $\neg(A) \rightarrow(O)$ is not provable, i.e.

$$
\neg(\neg \forall x(S x \rightarrow P x) \rightarrow \exists x(S x \wedge \neg P x)) Y_{i} \perp
$$

${ }^{5}$ In this context, a "gap" can mean a blank, a missing value. 
Proof

$$
\begin{gathered}
\frac{?(\neg(\neg \forall x(S x \rightarrow P x) \rightarrow \exists x(S x \wedge \neg P x)) \rightarrow \perp)}{\neg(\neg \forall x(S x \rightarrow P x) \rightarrow \exists x(S x \wedge \neg P x))} \\
\frac{? \perp}{?(\neg \forall x(S x \rightarrow P x) \rightarrow \exists x(S x \wedge \neg P x))} \\
\neg \forall x(S x \rightarrow P x) \\
\frac{? \exists x(S x \wedge \neg P x)}{S a} \\
\overbrace{P P a}^{? P x \rightarrow P x) \checkmark} \\
\times \quad \frac{? \neg P a}{P a}
\end{gathered}
$$

Therefore, according to Definition 2.2 the formula

$$
\neg(\neg \forall x(S x \rightarrow P x) \rightarrow \exists x(S x \wedge \neg P x))
$$

is a contradiction in CFOL but not in IFOL.

It seems that the previous proof could be a logical argument against intuitionistic logic which would suffer of a sort of deficiency. But the embarrassment that theorem 2.3 can cause is dissipated by the following one:

Theorem 2.4 A formula $F$ is valid in CFOL if and only its Kuroda translation $F^{k}$ is valid in IFOL.

Proof See David et al. [3, pp. 155-157]

There would be a real "intuitionistic gap in contradiction" only if there would be no intuitionistic translation of these formulas that are contradictory in CFOL but not IFOL. But we know that Godel-Gentzen translation or Kuroda translation are secure means to translate any formula valid in CFOL into a formula valid in IFOL, ${ }^{6}$ and therefore, any contradictory formula in CFOL can be translated by another contradictory formula in IFOL. For example, via Kuroda translation, the formula

$$
\neg \forall x(S x \rightarrow P x) \rightarrow \exists x(S x \wedge \neg P x)
$$

becomes

$$
\neg \neg(\neg \forall x \neg \neg(S x \rightarrow P x) \rightarrow \exists x(S x \wedge \neg P x))
$$

${ }^{6}$ See David et al. [3, p. 156] and von Plato [5, p. 170]. 
Therefore the translation of the classical contradiction (2.11) is in IFOL the negation of (2.13) and the translation of (2.9) is

$$
\neg(\neg \forall x \neg \neg(S x \rightarrow P x) \rightarrow \exists x(S x \wedge \neg P x)) \vdash_{i} \perp
$$

Regarding the intuitionistic square, it is therefore possible to translate every valid implication of the classical square into its Kuroda expression, valid in turn in IFOL, and then

- The three equivalences involved by the contradictory relations and lost in IFOL are, thanks to Kuroda translation, redefined as follows:

$$
\begin{aligned}
& \vdash_{i} \neg \neg(\forall x \neg \neg(S x \rightarrow P x) \leftrightarrow \neg \exists x(S x \wedge \neg P x)) \\
& \vdash_{i} \neg \neg(\exists x(S x \wedge \neg P x) \leftrightarrow \neg \forall x \neg \neg(S x \rightarrow P x)) \\
& \vdash_{i} \neg \neg(\exists x(S x \wedge P x) \leftrightarrow \neg \forall x \neg \neg(S x \rightarrow \neg P x))
\end{aligned}
$$

- once transformed via Kuroda translation, sub-contrary relation $\neg(O) \rightarrow(I)$ is valid in IFOL:

$$
\vdash_{i} \neg \neg((\neg x \neg \neg(S x \wedge \neg P x) \wedge \exists x(S x)) \rightarrow \exists x(S x \wedge P x))
$$

Consequently, nothing of the classical square of opposition is really lost in IFOL. But it remains to show in conclusion why Kuroda's translation is far from trivial and why some classical implications in the square of opposition are no longer valid in intuitionistic logic.

\section{Conclusion: What the Intuitionistic Square of Opposition Means Exactly}

\subsection{Kuroda Translation of the Classical Square}

As David et al. [3, p. 156] masterfully point out, Kuroda translation is a rigorous expression, from an intuitionistic point of view, of the specificity of classical logic. Because this translation shows the sufficient conditions to prove a formula $F$ in classical logic: 
Table 2 The intuitionistic amputations inside the classical square

$$
\begin{aligned}
& \nvdash_{i} \neg \forall x(S x \rightarrow P x) \rightarrow \exists x(S x \wedge \neg P x) \\
& \text { Proof. } \\
& \frac{?(\neg \forall x(S x \rightarrow P x) \rightarrow \exists x(S x \wedge \neg P x))}{\neg \forall x(S x \rightarrow P x)} \\
& ? \exists x(S x \wedge \neg P x) \\
& ?(S a \wedge \neg P a) \\
& \frac{? \forall x(S x \rightarrow P x)}{\frac{?(S b \rightarrow P b)}{S b}} \\
& ? P b \\
& Y_{i} \neg \forall x(S x \rightarrow \neg P x) \rightarrow \exists x(S x \wedge P x) \\
& \text { Proof. } \\
& \begin{aligned}
\frac{?(\neg \forall x(S x \rightarrow \neg P x) \rightarrow \exists x(S x \wedge P x))}{} & \\
& ? \exists x(S x \rightarrow P x \wedge P x)
\end{aligned} \\
& ?(S a \wedge P a) \\
& \frac{? \forall x(S x \rightarrow \neg P x) \checkmark}{\frac{?(S b \rightarrow \neg P b) \checkmark}{S b}} \\
& \frac{? \neg P b}{P b} \\
& \nvdash_{i}(\neg \exists x(S x \wedge \neg P x)) \rightarrow(\forall x(S x \rightarrow P x)) \\
& \nvdash_{i}(\neg \exists x(S x \wedge \neg P x) \wedge \exists x(S x)) \rightarrow \exists x(S x \wedge P x) \\
& \text { Proof. } \\
& \text { Proof. } \\
& \frac{?((\neg \exists x(S x \wedge \neg P x)) \rightarrow(\forall x(S x \rightarrow P x))) \checkmark}{\neg \exists x(S x \wedge \neg P x)} \\
& \frac{? \forall(S x \rightarrow P x) \checkmark}{\frac{?(S a \rightarrow P a)}{S a}} \\
& \text { ?Pa } \\
& ? \exists x(S x \wedge \neg P x) \\
& ?(S a \wedge \neg P a) \\
& \text { ?Sa } \frac{? \neg P a \checkmark}{P a} \\
& \frac{?((\neg \exists x(S x \wedge \neg P x) \wedge \exists x(S x)) \rightarrow \exists x(S x \wedge P x)) \checkmark}{\exists x(S x) \checkmark} \\
& \mathrm{Sa} \\
& \neg \exists x(S x \wedge \neg P x) \\
& ? \exists x(S x \wedge \neg P x) \\
& ? \exists x(S x \wedge P x) \\
& \overbrace{\substack{? S a \\
\times}}^{? P a} \frac{? \neg P a}{P a}
\end{aligned}
$$

1. using the rule of reductio ad absurdum on all sub-formulas of $F$ preceded by $\forall$ and on $F$ itself. $^{7}$

2. making use of the Ex Contradictione Quodlibet rule i.e the intuitionistic rule $\perp E^{8}$

\subsection{The Intuitionistic Amputations in the Classical Square}

By using Bell et al.'s proof method for IFOL, I am going to try to sum up the logical reasons of intuitionistic amputations in the classical square. The four classical implications in the square that are not provable in IFOL are contained Table 2 with their respective trees

\footnotetext{
${ }^{7}$ Hence Kuroda's translation recipe: put a double negation just before $F$ and just before each scope of universal quantifiers in $F$, e.g. if $\forall x(F x)$ is in $F$, it is translated by $\forall x \neg \neg(F x)$.

${ }^{8} \mathrm{Kuroda}$ 's translation is all what one needs as faithful translation of CFOL into IFOL. Godel-Gentzen translation goes further because contrary to the former, it translates also classical logic into minimal logic, see [3, pp. 157-158] .
} 
as countermodels. ${ }^{9}$

These amputations can be divided in two couples of formulas:

1. The trees of the pair $\{\neg(A) \rightarrow(O), \neg(E) \rightarrow(I)\}$ are countermodels showing that the assumption of the falsity of a universal statement does not analytically involve a counterexample disproving this latter.

2. The trees of the second pair, $\{\neg(O) \rightarrow(A), \neg(O) \rightarrow(I)\}$, are countermodels corresponding in IFOL to the intuitionistic refusal of reducing any double negation to a positive assertion.

The fact that these classical implications are no longer provable in IFOL can be explained by the independence of the connectives and quantifiers in intuitionistic logic, therefore that does not show that intuitionistic logic is weaker than classical logic, but that the former has stronger means of expressions than the latter.

\section{Acknowledgements Many thanks to}

- Baptiste Meles both for the discussions we had about the intuitionistic version of the square of opposition and for his very useful remarks on a draft of my paper,

- Jean-Yves Béziau who gave to me the occasion of writing this paper,

- My institution, the Archives Poincaré, which funded my participation to the fourth conference on the square of opposition (Vatican, May 5-9, 2014),

- the referees of the final version of this paper.

\section{References}

1. J. Bell, G. Solomon, D. DeVidi, Logical Options: An Introduction to Classical and Alternative Logics (Broadview Press, Peterborough, 2001)

2. J.-Y. Béziau, D. Jacquette (eds.), Around and Beyond the Square of Opposition (Studies in Universal Logic, Birkhaüser, 2012)

3. R. David, K. Nour, C. Raffalli, Introduction à la Logique: Théorie de la Démonstration (Dunod, Paris, 2004)

4. B. Méles, No group of opposition for constructive logics: the intuitionistic and linear cases, in Around and Beyond the Square of Opposition, ed. by J.-Y. Béziau, D. Jacquette (Studies in Universal Logic, Birkhaüser, 2012), pp. 201-218

5. J. von Plato, Elements of Logical Reasoning (Cambridge University Press, Cambridge, 2013)

J. Vidal-Rosset (四)

Philosophy Department, Lorraine University, Archives Poincaré - UMR 7117 - CNRS,

91 bd Libération, 54000 Nancy, France

e-mail: joseph.vidal-rosset@univ-lorraine.fi

\footnotetext{
${ }^{9}$ To reply to a question asked by a referee, it is indeed possible to see this tree method for intuitionistic logic as an automated search of Kripke countermodels. A formula $F$ is intuitionistically proved if and only if its refutation tree shows that it is not possible to give a Kripke countermodel for $F$, i.e. that the assumption that $F$ is intuitionistically unprovable (i.e. ?F) leads to a contradiction.
} 